\title{
Description of two new species of the genus Fimbristylis Vahl. (Cyperaceae) from Velliangiri Hills, Nilgiri Biosphere Reserve, India
}

\author{
M. Murugesan ${ }^{1}$, V. Balasubramaniam ${ }^{2} \&$ N. Nagarajan ${ }^{3}$ \\ ${ }^{1}$ Environmental Impact Assessment Division, Sálim Ali Centre for Ornithology and Natural History (SACON), Anaikatty (PO), \\ Coimbatore, Tamil Nadu 641108, India \\ ${ }^{2,3}$ PG and Research Department of Botany, Kongunadu Arts and Science College, G.N. Mills (PO), Coimbatore, Tamil Nadu 641029, \\ India \\ Email: ${ }^{1}$ taxonmurugesh@gmail.com, ${ }^{2}$ vbalu61@yahoo.co.in, ${ }^{3}$ nnagaraj7@yahoo.com
}

Abstract: We describe two new species of the genus Fimbristylis Vahl. (Cyperaceae) viz. Fimbristylis matthewii and F. velliangiriensis from Velliangiri hills in the Nilgiri Biosphere Reserve located in the Western Ghats area of Coimbatore District, Tamil Nadu, India. F. matthewii is close to F. uliginosa Steud. but differs by the presence of glabrous culms, much thickened culmbase by imbricating old leaf-sheaths, inflorescence terminal and subterminal or pseudolateral; involucral bracts 3-5; spikelets 2-9 (10) together, 15-30 flowered; glumes aristate; arista 0.6-1.2 mm long, sparsely scaberulous on the upper half of upper surface, and on nerves of the keels; nuts sparsely and minutely tuberculate. $F$. velliangiriensis is close to $F$. insignis Thw. but differs by the presence of leaves involute, acute at apex; spikelets 0.4-0.8 $\mathrm{cm}$ long; involucral bracts $3-5$, rarely 8 , equal to or longer than spikelets; glumes minutely scaberulous on upper surface, and aristate at apex; anthers with 2-8 ciliate hairy at tip; filaments broadly winged, often with a reddish gland at base; style winged; nut obovoid.

Keywords: Cyperaceae, Fimbristylis matthewii sp. nov. Fimbristylis velliangiriensis sp. nov., India, new species, Tamil Nadu, Velliangiri Hills.

Date of publication (online): 26 December 2010

Date of publication (print): 26 December 2010

ISSN 0974-7907 (online) | 0974-7893 (print)

Editor: Raju Sekar

Manuscript details:

Ms \# 02422

Received 11 March 2010

Final received 19 July 2010

Finally accepted 27 October 2010

Citation: Murugesan, M., V. Balasubramaniam \& N. Nagarajan (2010). Description of two new species of the genus Fimbristylis Vahl. (Cyperaceae) from Velliangiri Hills, Nilgiri Biosphere Reserve, India. Journal of Threatened Taxa 2(13): 1379-1381.

Copyright: (c) M. Murugesan, V. Balasubramaniam \& N. Nagarajan 2010. Creative Commons Attribution 3.0 Unported License. JoTT allows unrestricted use of this article in any medium for non-profit purposes, reproduction and distribution by providing adequate credit to the authors and the source of publication.

Acknowledgements: Authors thank Dr. G.V.S. Murthy, Joint Director Botanical Survey of India (MH), Southern Circle, Coimbatore for permission to consult the Herbarium specimens; Dr. M. Aruchami, Secretary and Dr. A.A. Siva Kumar, Principal of Kongunadu Arts and Science College, Coimbatore for facilities and encouragement. Our sincere thanks to Dr. V.B. Hosagoudar, Senior Scientist, Tropical Botanic Garden \& Research Institute, Palode, Thiruvananthapuram, Kerala for Latin diagnosis.

OPEN ACCESS I FREE DOWNLOAD (C) (i) (4)
The genus Fimbristylis Vahl. (Cyperaceae) has ca. 300 species distributed in tropical and subtropical regions with some in warmer parts of temperate regions. Species abundance is more in tropical Asia (Prasad \& Singh 2002). In India, 117 species have been reported (Prasad \& Singh 2002) with 53 species in Tamil Nadu (Henry et al. 1989).

During the floristic survey conducted between the years 2003-2008, in Velliangiri Hills (1840m 6040'$\left.7^{0} 10^{\prime} \mathrm{E} \& 10^{\circ} 55^{\prime}-11^{0} 10^{\prime} \mathrm{N}\right)$, two new species of the family Cyperaceae collected are described as new to science. The type specimens are in the Herbarium of Kongunadu Arts and Science College (KASCH), Coimbatore, and Madras Herbarium (MH), Botanical Survey of India, Southern Circle, Coimbatore, India.

\section{Fimbristylis matthewii M. Murugesan, V. Balasubramaniam \& N. Nagarajan, sp. nov.} (Fig. 1.)

Type: 03.vi.2004, Velliangiri Hills, Coimbatore District, Tamil Nadu, India, ca. 1800m, coll. Murugesan, KASCH 528 A-D, (Holotype-KASCH; Isotype-MH).

Etymology: The species is named after Dr. K.M. Matthew, angiosperm taxonomy expert of St. Joseph College, Trichirapalli, Tamil Nadu, India

Fimbristylis matthewii sp. nov. F. uliginosa Steud. afffinis, sed differt culmi glabri, crassus ad basim vagina foliorum imbricatus, inflorescentia terminalis et subterminalis vel pseudolateralis; bracteae involucrae 3-5; spicula tot 2-9 (10), 15-30-floribus; glumae aristatae; arista 0.6-1.2 mm longa, sparsim scaberulosae ad supra medium et nervis ad carina; nucis sparsim et minute tuberculatus.

Fimbristylis matthewii sp. nov. is closely allied to F. uliginosa Steud. but differs by the presence of glabrous culms, much thickened culm-base by imbricating old leaf-sheaths, inflorescence terminal and subterminal or pseudolateral; involucral bracts 3-5; spikelets 2-9 (10) together, 15-30- flowered; glumes aristate; arista 0.6-1.2 $\mathrm{mm}$ long, sparsely scaberulous on upper half of the upper surface, and on nerves of the keels; nuts sparsely and 


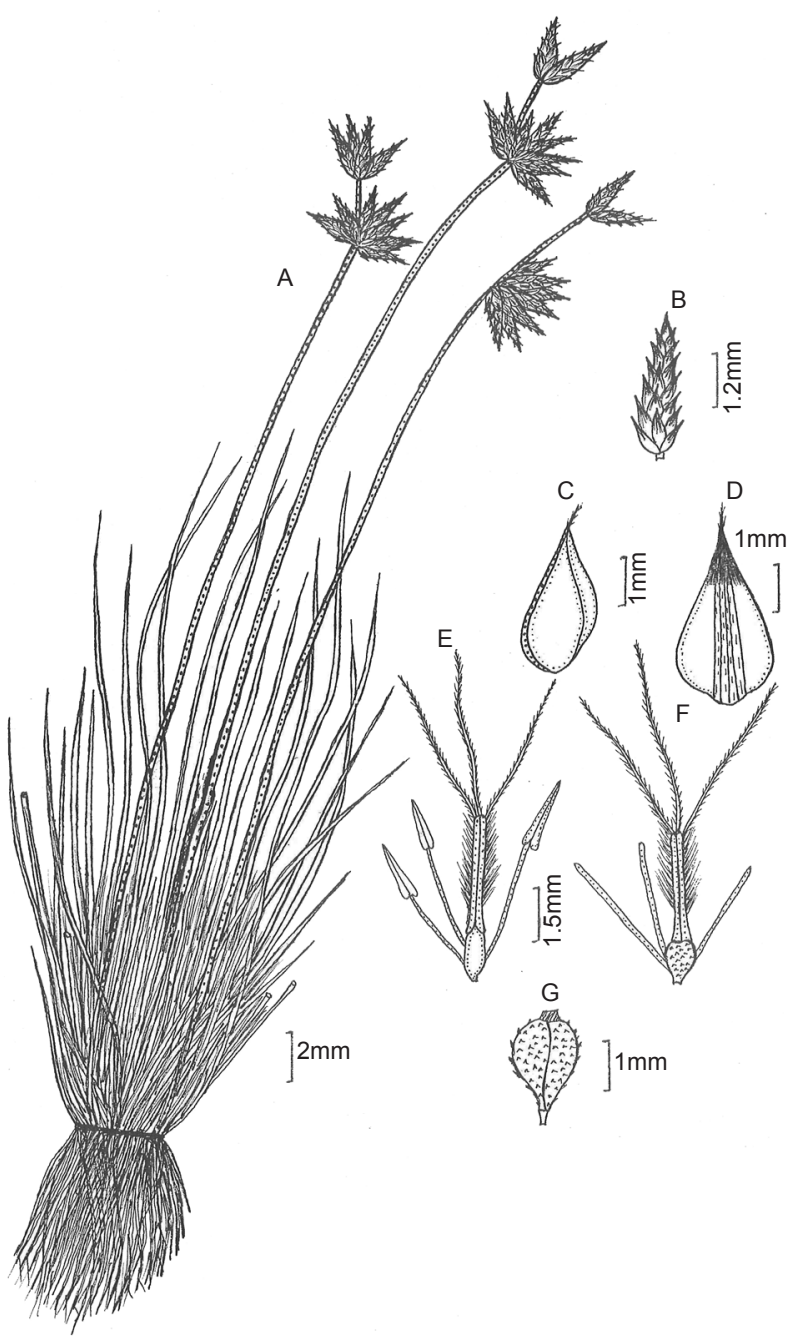

Figure 1. Fimbristylis matthewii sp. nov.

A - habitat; B - spikelet; C,D - glume; E,F - pistil; G - nut

minutely tuberculate.

Perennial, erect slender herb, $25-50 \mathrm{~cm}$ high; culms densely tufted, glabrous, 4-5 angled, much thickened at base by old leaf-sheaths; rhizome short, woody; roots wiry. Leaf half as long as the culms, linear, filiform, sometimes convoluted at margins, rarely narrowed at base, acute to shortly acuminate at apex, $7-25 \times 0.1$ $0.2 \mathrm{~cm}$, distantly scabrid along margins at base, closer towards apex; sheath blackish or brownish, 2-5 cm long, glabrous, breaking into fibers at maturity. Inflorescence in compound to decompound, terminal and subterminal or pseudolateral umbellate heads; involucral bracts 3-5, setaceous from a broad base, $0.5-2.5 \mathrm{~cm}$ long, scabrid at base and sharply spinulose scabrid towards apex, shortly and suddenly pointed at apex, prominently-faintly 1-nerved. Spikelets 2-9(10) together, all sessile, oblongellipsoid, reddish-brown, clustered into a head, 0.7-1.2 $\mathrm{x}$ 0.2-0.5 cm, 15-30-flowered. Glumes imbricate, ovate or oblong, 3.5-4 x 2-2.5 mm, 5-9-nerved, reddish-brown, narrowly scarious at margins, keeled, keels produced a small arista at apex; arista 0.6-1.2 mm long; sparsely, minutely scaberulous on nerves of the keel and upper surface of the glume in apical half. Rachilla prominently winged. Stamens 3; filaments linear, subequal, 1.8-3.5 $\mathrm{mm}$ long; anthers linear-oblong, 1.5-2 mm long, sagittate or cordate at base, acute or apiculate at apex. Ovary trigonous, obovate, 1-1.2 $\times 0.7-0.9 \mathrm{~mm}$. Style trigonous, slightly dilated pyramidal at base, $2.5-3 \mathrm{~mm}$ long, ciliate above middle and very dense at apex; stigmas 3 , ciliate, 1.5-2.5 mm long, shorter than style. Nut broadly obovoid, trigonous, $1-1.2 \times 0.8-1 \mathrm{~mm}$, umbonulate at apex, shortly stalked, sparsely and minutely tubercled, blackish or brownish.

Ecology: Rarely found in open grasslands at Velliangiri top at about $1800 \mathrm{~m}$ above $\mathrm{msl}$ along with Anaphalis beddomei, Arundinella purpurea, A. setosa, Ceropegia pusilla, Gynura travancorica, Jansenella griffithiana, Tripogon ananthaswamianus, T. bromoides, T. wightii and Valeriana arnottiana.

Flowering \& Fruiting: June-September

\section{Fimbristylis velliangiriensis M. Murugesan, V. Balasubramaniam \& N. Nagarajan, sp. nov.} (Fig. 2.)

Type: 29.x.2003, Velliangiri Hills, Coimbatore District, Tamil Nadu, India, ca. $1300 \mathrm{~m}$, coll. Murugesan, $\mathrm{KASCH}$ 359 A-D, (Holotype-KASCH; Isotype-MH).

Etymology: After the type locality Velliangiri Hills.

Fimbristylis velliangiriensis $F$. insignis Thw. afffinis, sed differt folia involuta, ad apicem acuta; spicula 0.4-0.8 cm longa; bracteae involucrae 3-5, raro 8, equantiae vel spicula longioribus; glumae minute scaberulae ad pagina superiorum et ad apicem aristatae; antherae ad apicem 2-8 ciliatae; filium late alarum, saepe glandula ruber ad basim; styli alati; nucis obovoideus.

Fimbristylis velliangiriensis is closely allied to $F$. insignis Thw. but differs by the presence of leaves involute, acute at apex; spikelets $0.4-0.8 \mathrm{~cm}$ long; involucral bracts $3-5$, rarely 8 , equal to or longer than spikelets; glumes minutely scaberulous on upper surface, and aristate at apex; anthers with 2-8 ciliate hairy at tip; filaments broadly winged, often with a reddish gland at base; style winged; nut obovoid.

Perennial, erect slender herb, $20-35 \mathrm{~cm}$ high; rhizome absent or often very short, woody; roots wiry. Culm(s) solitary, sometimes tufted, smooth or often scabrid, 4-5 angled, much thickened at base by old leaf-sheaths. Leaf half as long as or often $2 / 3$ of the stem, linear, 3-5-nerved, involute, scabrid along margins, suddenly and shortly pointed at apex, 5-18 x 0.1-0.3 cm; sheaths hyaline, whitish or brownish, $1.2-4 \mathrm{~cm}$ long, glabrous, breaking into fibers when maturity. Inflorescence in terminal compound to decompound, anthelate, $2.5-4 \times 1-2.5 \mathrm{~cm}$, 9-15- spikeleted. Spikelets ellipsoid or oblongish, 0.4-0.8 


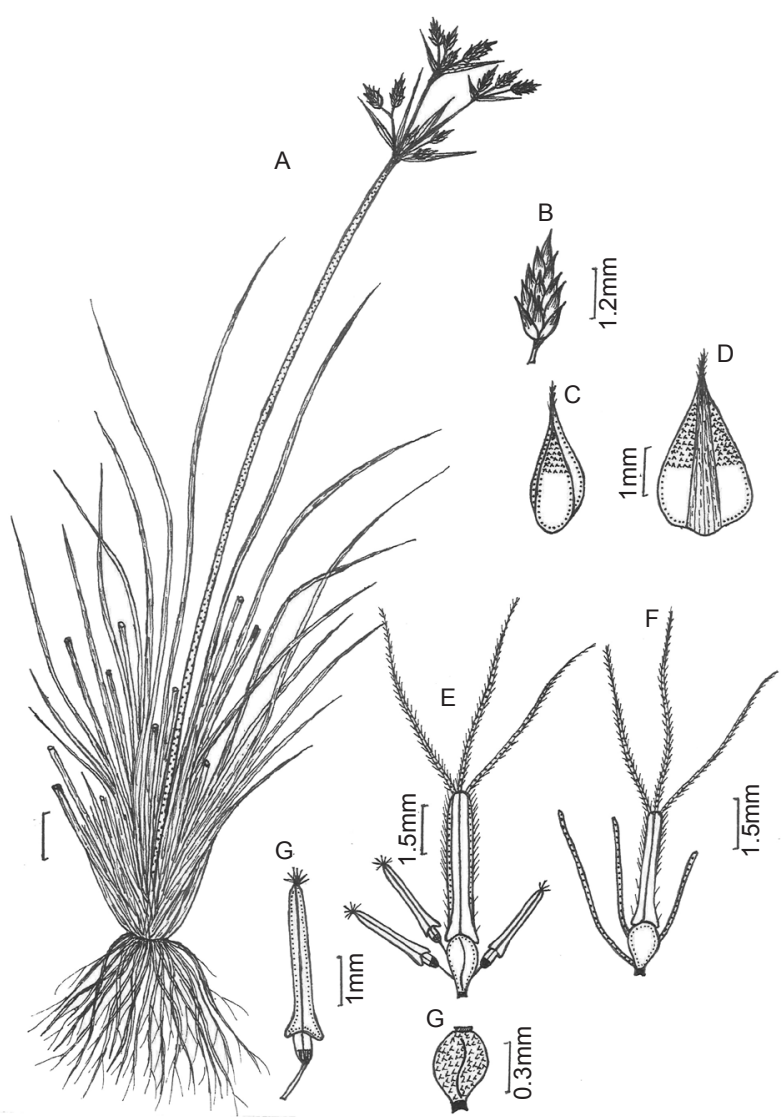

Figure 2. Fimbristylis velliangiriensis sp. nov. A - habitat; B - spikelet; C,D - glume; E,F - pistil; G - stamen; $\mathrm{H}$ - nut

cm long, reddish-brown, pedicelled, rarely 1 or 2 spikelets sessile, 6-10(13) flowered; pedicel 2.5-7.5 mm long; involucral bracts $3-5$, rarely $8,0.8-2 \mathrm{~cm}$ long, equal to or longer than spikelets, setaceous from broad base, scabrid at base, sharply spinulose scabrid towards apex, shortly and suddenly pointed at apex. Rachilla narrowly winged.
Glumes imbricate, broadly ovate-oblong, 3.5-4 x 2.5-3.3 $\mathrm{cm}$, reddish-brown, 7-9- nerved, keeled; keels produced into a arista at apex; arista 1.0-1.2 mm long, margins narrowly hyaline or scarious, minutely and scatteredly scaberulous without. Stamens 3-3.5 mm long; filaments broadly winged on both sides flat, subequal, 0.7-1.1 mm long, rarely reddish glandular at base; anthers linearoblong or oblong-lanceolate, 2-3 mm long, 2-8 ciliate hairy at apex, sagittate at base and shortly winged at base. Ovary trigonus, obovate, 0.5-0.8 mm long, slightly winged on angles. Style trigonus, $2.5-3.5 \mathrm{~mm}$ long, prominently winged on the angle, glabrous at base, densely ciliate above the middle; stigmas 3, 2.5-4 mm long, densely ciliate along margins, equal to or longer then style. Nut trigonous, obovoid, 0.8-1.2 mm long, minutely tubercled.

Ecology: Common in open grasslands at about $1300 \mathrm{~m}$ along with Arundinella pumila, A. purpurea var. purpurea, Biophytum sensitivum, Fimbristylis uliginosa, Swertia angustifolia, S. corymbosa, Tripogon ananthaswamianus and T. bromoides.

Flowering \& Fruiting: September-November.

\section{REFERENCES}

Gamble, J.S. \& C.E.C. Fischer (1915-1936). The Flora of the Presidency of Madras. Part 1-11, Vol. 3. (Part 1-7 by Gamble and 8-11 by Fischer). Adlard \& Sons Ltd., London, 16481660pp. (Repr. ed. Vols.1-3.1957).

Henry, A.N., V. Chitra \& N.P. Balakrishnan (1989). Flora of Tamil Nadu, India. Ser. 1: Analysis. vol. 3. Botanical Survey of India, Coimbatore, 77-82pp.

Hooker, J.D. (ed.) (1897). The Flora of British India Vol. 6. Reeve \& Co., London, 630-651pp.

Prasad, V.P. \& N.P. Singh (2002). Sedges of Karnataka, India. (Family-Cyperaceae). Journal of Economic and Taxonomic Botany, Additional series 21: 1-337.

Rao, A.S. \& D.M. Verma (1982). Cyperaceae of North East India. Botanical Survey of India, Howrah, 23-54pp. 